\title{
Homomorphic Pattern Mining from a Single Large Data Tree
}

\author{
Xiaoying $\mathrm{Wu}^{1} \cdot$ Dimitri Theodoratos ${ }^{2}$
}

Received: 6 July 2016/ Accepted: 19 December 2016/Published online: 10 January 2017

(C) The Author(s) 2017. This article is published with open access at Springerlink.com

\begin{abstract}
Finding interesting tree patterns hidden in large datasets is a central topic in data mining with many practical applications. Unfortunately, previous contributions have focused almost exclusively on mining-induced patterns from a set of small trees. The problem of mining homomorphic patterns from a large data tree has been neglected. This is mainly due to the challenging unbounded redundancy that homomorphic tree patterns can display. However, mining homomorphic patterns allows for discovering large patterns which cannot be extracted when mining induced or embedded patterns. Large patterns better characterize big trees which are important for many modern applications in particular with the explosion of big data. In this paper, we address the problem of mining frequent homomorphic tree patterns from a single large tree. We propose a novel approach that extracts non-redundant maximal homomorphic patterns. Our approach employs an incremental frequency computation method that avoids the costly enumeration of all pattern matchings required by previous approaches. Matching information of already computed patterns is materialized as bitmaps, a technique that not only minimizes the memory consumption, but also the CPU time. Our contribution also includes an
\end{abstract}

The research of Wu was supported by the National Natural Science Foundation of China under Grant Nos. 61202035 and 61272110.

Dimitri Theodoratos

dth@njit.edu

Xiaoying Wu

xiaoying.wu@whu.edu.cn

1 State Key Laboratory of Software Engineering, Wuhan University, Wuhan, China

2 New Jersey Institute of Technology, Newark, NJ, USA optimization technique which can further reduce the search space of homomorphic patterns. We conducted detailed experiments to test the performance and scalability of our approach. The experimental evaluation shows that our approach mines larger patterns and extracts maximal homomorphic patterns from real and synthetic datasets outperforming state-of-the-art embedded tree mining algorithms applied to a large data tree.

\section{Introduction}

Extracting frequent tree patterns which are hidden in data trees is central for analyzing data and is a base step for other data mining processes including association rule mining, clustering and classification. Trees have emerged in recent years as the standard format for representing, exporting, exchanging and integrating data on the web (e.g., XML and JSON). Tree data are adopted in various application areas and systems such as business process management, NoSQL databases, key-value stores, scientific workflows, computational biology and genome analysis.

Because of its practical importance, tree mining has been extensively studied [2, 3, 5, 6, 8-11, 14-19, 25-27]. The approaches to tree mining can be basically characterized by two parameters: (a) the type of morphism used to map the tree patterns to the data structure and (b) the type of mined tree data.

Mining homomorphic tree patterns The morphism determines how a pattern is mapped to the data tree. The morphism definition depends also on the type of pattern considered. In the literature, two types of tree patterns have been studied: patterns whose edges represent parent-child 
relationships (child edges) and patterns whose edges represent ancestor-descendant relationships (descendant edges). Over the years, research has evolved from considering isomorphisms for mining patterns with child edges (induced patterns) $[2,5]$ to considering embeddings for mining patterns with descendant edges (embedded patterns) $[17,26,27]$. Because of the descendant edges, embeddings are able to extract patterns "hidden" (or embedded) deep within large trees which might be missed by the induced pattern definition [26]. Nevertheless, embeddings are still restricted because: (a) They are injective (one-to-one), and (b) they cannot map two sibling nodes in a pattern to two nodes on the same path in the data tree. On the other hand, homomorphisms are powerful morphisms that do not have those two restrictions of embeddings. We term patterns with descendant edges, mined through homomorphisms, homomorphic patterns. Formal definitions are provided in Sect. 2. As homomorphisms are more relaxed than embeddings, the mined homomorphic patterns are a superset of the mined embedded patterns.

Figure 1a shows four data trees corresponding to different schemas to be integrated through the mining of large tree patterns. The frequency threshold is set to three. Figure $1 \mathrm{~b}$ shows induced mined tree patterns, embedded patterns and non-redundant homomorphic patterns. Figure $1 \mathrm{~b}$ includes the largest patterns that can be mined in each category. As one can see, the shown embedded patterns are not induced patterns, and the shown homomorphic patterns are neither embedded nor induced patterns. Further, the homomorphic patterns are larger than all the other patterns.

Large patterns are more useful in describing data. Mining tasks usually attach much greater importance to patterns that are larger in size, e.g., longer sequences are usually of more significant meaning than shorter ones in bioinformatics [29]. As mentioned in [28], large patterns have become increasingly important in many modern applications.

Therefore, homomorphisms and homomorphic patterns display a number of advantages. First, they allow the extraction of patterns that cannot be extracted by embedded patterns. Second, extracted homomorphic patterns can be larger than embedded patterns. Finally, homomorphisms can be computed more efficiently than embeddings. Indeed, the problem of checking the existence of a homomorphism of an unordered tree pattern to a data tree is polynomial [13], while the corresponding problem for an embedding is NP-complete [12].

Mining patterns from a large data tree The type of mined data can be a collection of small trees $[2,5,17,26,27]$ or a single large tree. Surprisingly, the problem of mining tree patterns from a single large tree has only very recently been touched even though a plethora of interesting datasets from different areas are in the form of a single large tree. Examples include encyclopedia databases like Wikipedia, bibliographic databases like PubMed, scientific and experimental result databases like UniprotKB, and biological datasets like phylogenetic trees. These datasets grow constantly with the addition of new data. Big data applications seek to extract information from large datasets. However, mining a single large data tree is more complex than mining a set of small data trees. In fact, the former setting is more general than the latter, since a collection of small trees can be modeled as a single large tree rooted at a virtual unlabeled node. Existing algorithms for mining embedded patterns from a collection of small trees [26] cannot scale well when the size of the data tree increases. Our experiments show that these algorithms cannot scale beyond some hundreds of nodes in a data tree with low-frequency thresholds.

The problem Unfortunately, previous work has focused almost exclusively on mining-induced and embedded patterns from a set of small trees. The issue of mining homomorphic patterns from a single large data tree has been neglected.

The challenges Mining homomorphic tree patterns is a challenging task. Homomorphic tree patterns are difficult to handle as they may contain redundant nodes. If their structure is not appropriately constrained, the number of frequent patterns (and therefore the number of candidate patterns that need to be generated) can be infinite.

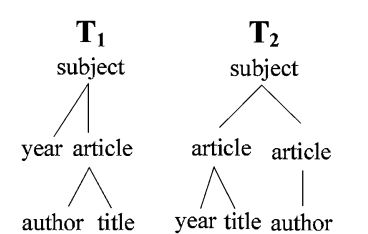

(a)

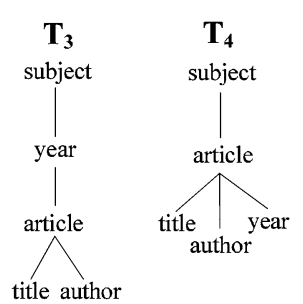

)
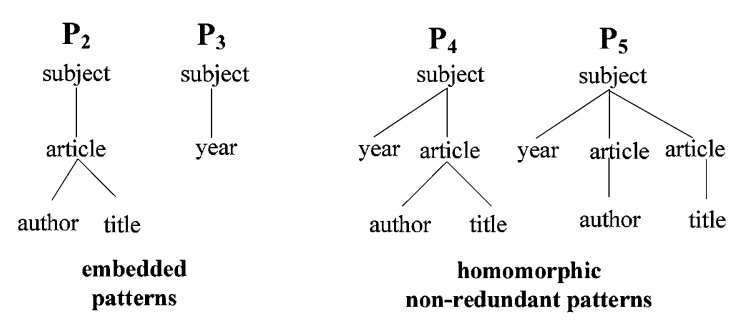

(b)

Fig. 1 Different types of mined tree patterns occurring in three of the four data trees. a Data trees, $\mathbf{b}$ mined tree patterns 
Even if homomorphic patterns are successfully constrained to be non-redundant, their number can be much larger than that of frequent embedded patterns from the same data tree. In order for the mining algorithm to be efficient, new, much faster techniques for computing the support of the candidate homomorphic tree patterns need to be devised.

The support of patterns in the single large data tree setting cannot be anymore the number of trees that contain the pattern as is the case in the multiple small trees setting. A new way to define pattern support in the new setting is needed which enjoys useful monotonic characteristics.

Typically, one can deal with a large number of frequent patterns, by computing only maximal frequent patterns. In the context of induced tree patterns, a pattern is maximal if there is no frequent superpattern [5]. A non-maximal pattern is not returned to the user as there is a larger, more specific pattern, which is frequent. However, in the context of homomorphic patterns, which involve descendant edges, the concept of superpattern is not sufficient for capturing the specificity of a pattern. A tree pattern can be more specific (and informative) without being a superpattern. For instance, the homomorphic pattern $P_{4}$ of Fig. $1 \mathrm{~b}$ is more specific than the homomorphic pattern $P_{5}$ without being a superpattern of $P_{5}$. Therefore, a new sophisticated definition for maximal patterns is required which takes into account both the particularities of the homomorphic patterns and the single large tree setting.

Contribution In this paper, we address the problem of mining maximal homomorphic unordered tree patterns from a single large data tree. Our main contributions are:

- We define the problem of extracting homomorphic and maximal homomorphic unordered tree patterns with descendant relationships from a single large data tree. This problem departs from previous ones which focus on mining-induced or embedded tree patterns from a set of small data trees.

- We constrain the extracted homomorphic patterns to be non-redundant in order to avoid dealing with an infinite number of frequent patterns of unbounded size. In order to define maximal patterns, we introduce a strict partial order on patterns characterizing specificity. A pattern which is more specific provides more information on the data tree.

- We design an efficient algorithm to discover all frequent maximal homomorphic tree patterns. Our algorithm wisely prunes the search space by generating and considering patterns that are maximal and frequent or can contribute to the generation of maximal frequent patterns. It also exploits an optimization technique which relies on pattern ordering to further reduce the space of homomorphic patterns.
- Our algorithm employs an incremental frequency computation method that avoids the costly enumeration of all pattern matchings required by previous approaches. An originality of our method is that matching information of already computed patterns is materialized as bitmaps. Exploiting bitmaps not only minimizes the memory consumption, but also reduces CPU costs.

- We run extensive experiments to evaluate the performance and scalability of our approach on real datasets. The experimental results show that: (a) The mined maximal homomorphic tree patterns are larger on the average than maximal embedded tree patterns on the same datasets, (b) our approach mines homomorphic maximal patterns up to several orders of magnitude faster than state-of-the-art algorithms mining embedded tree patterns when applied to a large data tree, (c) our algorithm consumes only a small fraction of the memory space and scales smoothly when the size of the dataset increases, and(d) the optimization technique substantially improves the time performance of the algorithm.

Paper outline The next section introduces various related concepts and formally defines the problem. Section 3 presents our algorithm that discovers all frequent maximal homomorphic tree patterns. Our comparative experimental results are presented and analyzed in Sect. 4. Related work is reviewed in Sect. 5. Section 6 concludes and suggests future work.

\section{Preliminaries and Problem Definition}

Trees and inverted lists We consider rooted labeled trees, where each tree has a distinguished root node and a labeling function $l b$ mapping nodes to labels. A tree is called ordered if it has a predefined left-to-right ordering among the children of each node. Otherwise, it is unordered. The size of a tree is defined as the number of its nodes. In this paper, unless otherwise specified, a tree pattern is a rooted, labeled, unordered tree.

For every label $a$ in an input data tree $T$, we construct an inverted list $L_{a}$ of the data nodes with label $a$ ordered by their pre-order appearance in $T$. Figure $2 \mathrm{a}, \mathrm{b}$ shows a data tree and inverted lists of its labels.

Tree morphisms There are two types of tree patterns: patterns whose edges represent child relationships (child edges) and patterns whose edges represent descendant relationships (descendant edges). In the literature of tree pattern mining, different types of morphisms are employed to determine whether a tree pattern is included in a tree. 


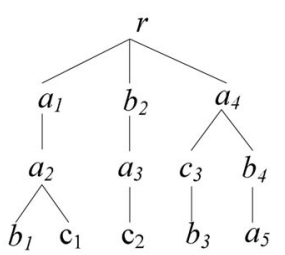

(a)

$$
\begin{aligned}
& \mathrm{L}_{\mathrm{a}}=\left\{\mathrm{a}_{1}, \mathrm{a}_{2}, \mathrm{a}_{3}, \mathrm{a}_{4}, \mathrm{a}_{5}\right\} \\
& \mathrm{L}_{\mathrm{b}}=\left\{\mathrm{b}_{1}, \mathrm{~b}_{2}, \mathrm{~b}_{3}, \mathrm{~b}_{4}\right\} \\
& \mathrm{L}_{\mathrm{c}}=\left\{\mathrm{c}_{1}, \mathrm{c}_{2}, \mathrm{c}_{3}\right\} \\
& \mathrm{L}_{\mathrm{r}}=\{\mathrm{r}\}
\end{aligned}
$$

\begin{tabular}{|c|c|c|c|c|}
\hline pattern & occurrences & occur. lists & bitmaps & sup. \\
\hline & $\begin{array}{r}\left\{a_{1} b_{1} c_{1}, a_{2} b_{1} c_{1},\right. \\
\left.a_{4} b_{3} c_{3}, a_{4} b_{4} c_{3}\right\}\end{array}$ & $\begin{array}{l}\mathrm{L}_{\mathrm{A}}=\left\{\mathrm{a}_{1}, \mathrm{a}_{2}, \mathrm{a}_{4}\right\} \\
\mathrm{L}_{\mathrm{B}}=\left\{\mathrm{b}_{1}, \mathrm{~b}_{3}, \mathrm{~b}_{4}\right\} \\
\mathrm{L}_{\mathrm{C}}=\left\{\mathrm{c}_{1}, \mathrm{c}_{3}\right\}\end{array}$ & $\begin{array}{l}\mathrm{L}_{\mathrm{A}}=11010 \\
\mathrm{~L}_{\mathrm{B}}=1011 \\
\mathrm{~L}_{\mathrm{C}}=101\end{array}$ & 2 \\
\hline
\end{tabular}

(b) (c)

Fig. 2 A tree $T$, its inverted lists and occurrence info. of pattern $P$ on $T$. a A tree $T$, b inverted lists, $\mathbf{c}$ occurrence information for pattern $P$ on tree $T$

Given a pattern $P$ and a tree $T$, a homomorphism from $P$ to $T$ is a function $m$ mapping nodes of $P$ to nodes of $T$, such that: (1) for any node $x \in P, l b(x)=l b(m(x))$; and (2) for any edge $(x, y) \in P$, if $(x, y)$ is a child edge, $(m(x), m(y))$ is an edge of $T$, while if $(x, y)$ is a descendant edge, $m(x)$ is an ancestor of $m(y)$ in $T$.

Previous contributions have constrained the homomorphisms considered for tree mining in different ways. Let $P$ be a pattern with descendant edges. An embedding from $P$ to $T$ is an injective function $m$ mapping nodes of $P$ to nodes of $T$, such that: (1) for any node $x \in P, l b(x)=l b(m(x))$; and (2) $(x, y)$ is an edge in $P$ iff $m(x)$ is an ancestor of $m(y)$ in $T$. Clearly, an embedding is also a homomorphism. Notice that, in contrast to a homomorphism, an embedding cannot map two siblings of $P$ to two nodes on the same path in $T$. Patterns with descendant edges mined using embeddings are called embedded patterns. We call patterns with descendant edges mined using homomorphisms homomorphic patterns. In this paper, we consider mining homomorphic patterns. The set of frequent embedded patterns on a data tree $T$ is a subset of the set of frequent homomorphic patterns on $T$ since embeddings are restricted homomorphisms.

Pattern nodes occurrence lists We identify an occurrence of $P$ on $T$ by a tuple indexed by the nodes of $P$ whose values are the images of the corresponding nodes in $P$ under a homomorphism of $P$ to $T$. The set of occurrences of $P$ under all possible homomorphisms of $P$ to $T$ is a relation $O C$ whose schema is the set of nodes of $P$. If $X$ is a node in $P$ labeled by label $a$, the occurrence list of $X$ on $T$ is a sublist $L_{X}$ of the inverted list $L_{a}$ containing only those nodes that occur in the column for $X$ in $O C$.

As an example, in Fig. 2c, the second and third columns give the occurrence relation and the node occurrence lists, respectively, of the pattern $P$ on the tree $T$ of Fig. 2a.

Support We adopt for the support of tree patterns root frequency: The support of a pattern $P$ on a data tree $T$ is the number of distinct images (nodes in $T$ ) of the root of $P$ under all homomorphisms of $P$ to $T$. In other words, the support of $P$ on $T$ is the size of the occurrence list of the root of $P$ on $T$.
A pattern $S$ is frequent if its support is no less than a user-defined threshold minsup. We denote by $F_{k}$ the set of all frequent patterns of size $k$, also known as a $k$ pattern.

Constraining patterns When homomorphisms are considered, it is possible that an infinite number of frequent patterns of unrestricted size can be extracted from a dataset. In order to exclude this possibility, we consider and define next non-redundant patterns. We say that two patterns $P_{1}$ and $P_{2}$ are equivalent, if there exists a homomorphism from $P_{1}$ to $P_{2}$ and vice-versa. A node $X$ in a pattern $P$ is redundant if the subpattern obtained from $P$ by deleting $X$ and all its descendants is equivalent to $P$. For example, the rightmost node $C$ of $P_{3}$ and the rightmost node $B$ of $P_{5}$ in Fig. 3 are redundant. Adding redundant nodes to a pattern can generate an infinite number of frequent equivalent patterns which have the same support. These patterns are not useful as they do not provide additional information on the data tree. A pattern is non-redundant if it does not have redundant nodes. In Fig. 3, patterns $P_{3}$ and $P_{5}$ are redundant, while the rest of the patterns are non-redundant. Non-redundant patterns correspond to minimal tree pattern queries [1] in tree databases. Their number is finite. We discuss later how to efficiently check patterns for redundancy by identifying redundant nodes. We set forth to extract only frequent patterns which are non-redundant, but in the process of finding frequent non-redundant patterns, we might generate also some redundant patterns.

Maximal patterns In order to define maximal homomorphic frequent patterns, we introduce a specificity relation on patterns: A pattern $P_{1}$ is more specific than a pattern $P_{2}$ (and $P_{2}$ is less specific than $P_{1}$ ) iff there is a homomorphism from $P_{2}$ to $P_{1}$ but not from $P_{1}$ to $P_{2}$. If a pattern $P_{1}$ is more specific than a pattern $P_{2}$, we write $P_{1} \prec P_{2}$. For instance, in Fig. 3, $P_{1} \prec P_{i}, i=2, \ldots, 7$, and $P_{2} \prec P_{6}$. Similarly, in Fig. 1, $P_{2} \prec P_{1}, P_{5} \prec P_{3}, P_{4} \prec P_{2}$ and $P_{4} \prec P_{5}$. Note that $P_{4}$ is more specific than $P_{5}$ even though it is smaller in size than $P_{5}$. Clearly, $\prec$ is a strict partial order. If $P_{1} \prec P_{2}, P_{1}$ conveys more information on the dataset than $P_{2}$. 
Fig. 3 A data tree and homomorphic patterns. a A data tree $T$, b Homomorphic patterns on $T$

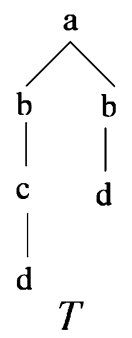

(a)
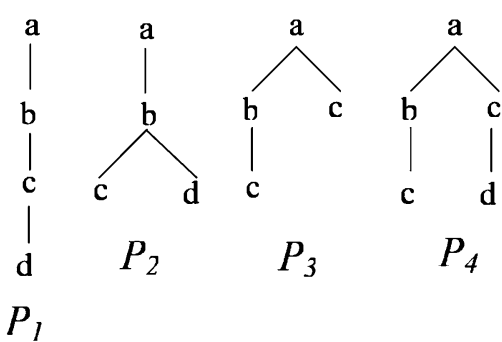

(b)
A frequent pattern $P$ is maximal if there is no other frequent pattern $P_{1}$, such that $P_{1} \prec P$. For instance, in Fig. 1, all the patterns shown are frequent homomorphic patterns and $P_{4}$ is the only maximal pattern.

Problem statement Given a large tree $T$ and a minimum support threshold minsup, our goal is to mine all maximal homomorphic frequent patterns from $T$.

\section{Proposed Approach}

Our approach for mining homomorphic tree patterns from a large tree iterates between the candidate generation phase and the support counting phase. In the first phase, we use a systematic way to generate candidate patterns that are potentially frequent. In the second phase, we develop an efficient method to compute the support of candidate patterns.

\subsection{Candidate Generation}

To generate candidate patterns, we adapt in this section the equivalence class-based pattern generation method proposed in $[26,27]$ so that it can address pattern redundancy and maximality. A candidate pattern may have multiple alternative isomorphic representations. To minimize the redundant generation of the isomorphic representations of the same pattern, we employ a canonical form for tree patterns [7].

\subsubsection{Equivalence Class-Based Pattern Generation}

Let $P$ be a pattern of size $k-1$. Each node of $P$ is identified by its depth-first position in the tree, determined through a depth-first traversal of $P$, by sequentially assigning numbers to the first visit of the node. The rightmost leaf of $P$, denoted $\mathrm{rml}$, is the node with the highest depth-first position. The immediate prefix of $P$ is the subpattern of $P$ obtained by deleting the $r m l$ from $P$. The equivalence class of $P$ is the set of all the patterns of size $k$ that have $P$ as their immediate prefix. We denote the equivalence class of $P$ as $[P]$. Any two members of $[P]$ differ only in their $r m l s$.
We use the notation $P_{x}^{i}$ to denote the $k$-pattern formed by adding a child node labeled by $x$ to the node with position $i$ in $P$ as the $r m l$.

Given an equivalence class $[P]$, we obtain its successor classes by expanding patterns in $[P]$. Specifically, candidates are generated by joining each pattern $P_{x}^{i} \in[P]$ with any other pattern $P_{y}^{j}$ in $[P]$, including itself, to produce the patterns of the equivalence class $\left[P_{x}^{i}\right]$. We denote the above join operation by $P_{x}^{i} \otimes P_{y}^{j}$. There are two possible outcomes for each $P_{x}^{i} \otimes P_{y}^{j}$ : One is obtained by making $y$ a sibling node of $x$ in $P_{x}^{i}$ (cousin expansion), the other is obtained by making $y$ a child node of $x$ in $P_{x}^{i}$ (child expansion). We call patterns $P_{x}^{i}$ and $P_{y}^{j}$ the left parent and right parent of a join outcome, respectively.

As an example, in Fig. 3, patterns $P_{1}, P_{2}, P_{3}, P_{5}$, and $P_{7}$ are members of class $[a / b / c] ; P_{4}$ is a join outcome of $P_{3} \otimes P_{7}$, obtained by making the $r m l d$ of $P_{7}$ a child of the rml c of $P_{3}$.

\subsubsection{Checking Pattern Redundancy}

The pattern generation process may produce candidates which are redundant (defined in Sect. 2). We discuss below how to efficiently check pattern redundancy by identifying redundant nodes. We exploit a result of [1] which states that: A node $X$ of a pattern $P$ is redundant iff there exists a homomorphism $h$ from $P$ to itself such that $h(X) \neq X$. A brute-force method for checking whether a pattern is redundant computes all the possible homomorphisms from $P$ to itself. Unfortunately, the number of the homomorphisms can be exponential on the size of $P$. Therefore, we have designed an algorithm called computeHoms which, given patterns $P$ and $Q$, compactly encodes all the homomorphisms from $P$ to $Q$ in polynomial time and space. This algorithm enhances the previous one presented in [13] which checks whether there exists a homomorphism from one tree pattern to another, while achieving the same time and space complexity.

Algorithm computeHoms Algorithm computeHoms is presented in Fig. 4. It deploys a standard dynamic programming technique for computing a Boolean matrix $\mathcal{M}(p$, 
Input: two patterns $P$ and $Q$.

Output: a Boolean matrix $\mathcal{M}$ that encodes all the homomorphisms from $P$ to $Q$.

1. Initialize a boolean matrix $\mathcal{C}(p, q), p \in \operatorname{nodes}(P), q \in \operatorname{nodes}(Q)$;

2. if $(\operatorname{BottomUpTraversal}(\mathcal{C}))$ then

3. $\mathcal{M}:=$ TopDownTraversal $(\mathcal{C})$;

4. else

5. there is no homomorphism from $P$ to $Q$;

Function BottomUpTraversal(Matrix $\mathcal{C}$ )

1. Initialize a boolean matrix $\mathcal{D}(p, q), p \in \operatorname{nodes}(P), q \in \operatorname{nodes}(Q)$;

2. for (every node $q$ of $Q$ in bottom-up order) do

3. for (every node $p$ of $P$ in bottom-up order) do

4. $\quad \mathcal{C}(p, q):=(l b(q)=l b(q)) \wedge$

$\bigwedge_{u \in \text { children }(p)}\left(\bigvee_{v \in \text { children }(q)} \mathcal{D}(u, v)\right)$;

5. $\mathcal{D}(p, q):=\mathcal{C}(p, q) \vee \bigvee_{v \in \text { children }(q)} \mathcal{D}(p, v)$;

6. return $\mathcal{D}(\operatorname{root}(p), \operatorname{root}(q))$;

Function TopDownTraversal(Matrix $\mathcal{C}$ )

1. Initialize two boolean matrices $\mathcal{P}(p, q)$ and $\mathcal{A}(p, q), p \in \operatorname{nodes}(P)$, $q \in \operatorname{nodes}(Q)$;

2. for (every node $q$ of $Q$ in top-down order) do

3. for (every node $p$ of $P$ in top-down order) do

4. $\mathcal{P}(p, q):=(\mathcal{C}(p, q)) \wedge \mathcal{A}($ parent of $p$, parent of $q) ;$

$\mathcal{A}(p, q):=\mathcal{P}(p, q) \vee \mathcal{A}(p$, parent of $q)$

6. return $\mathcal{P}$

Fig. 4 Algorithm computeHoms

$q), p \in \operatorname{nodes}(P), q \in \operatorname{nodes}(Q)$, such that $\mathcal{M}(p, q)$ is true if: (1) There exists a homomorphism from the subpattern rooted at $p$ to the subpattern rooted at $q$ (Function BottomUpTraversal); and (2) there exists a homomorphism from the prefix path of $p$ to the prefix path of $q$, where prefix path of a node is the path from the pattern root to that node (Function TopDownTraversal). Without loss of generality, we assume that both $P$ and $Q$ have a virtual root $r$. We now describe the algorithm in more detail.

The algorithm first performs a bottom-up traversal of $P$ and $Q$ (Function BottomUpTraversal) to compute a Boolean matrix $\mathcal{C}$. Entry $\mathcal{C}(p, q)$ is true if there exists a homomorphism from the subpattern rooted at $p$ to the subpattern rooted at $q$. To eliminate redundant computations, the bottom-up traversal also computes a second matrix $\mathcal{D}$. Entry $\mathcal{D}(p, q)$ is true if there exists a homomorphism from the subpattern rooted at $p$ to some subpattern of $Q$ whose root is either $q$ or a descendant of $q$.

If BottomUpTraversal returns true, the algorithm proceeds to perform a top-down traversal of $P$ and $Q$ (Function TopDownTraversal) to compute a Boolean matrix $\mathcal{P}$. Entry $\mathcal{P}(p, q)$ is true if $\mathcal{C}(p, q)$ (computed by the bottom-up traversal) is true and there exists a homomorphism from the prefix path of $p$ to the prefix path of $q$. As with the bottom-up traversal, a second matrix $\mathcal{A}$ is computed. Entry $\mathcal{A}(p, q)$ is true if there exists a homomorphism from the prefix path of $p$ to some prefix path of either $q$ or an ancestor of $q$.
Proposition 1 There exists a homomorphism from pattern $P$ to pattern $Q$ that maps node $p \in P$ to node $q \in Q$ iff entry $\mathcal{M}(p, q)$ is true, where $\mathcal{M}$ is the Boolean matrix computed by Algorithm computeHoms on $P$ and $Q$.

The proof of Proposition 1 is straightforward by the definition of pattern homomorphisms and the construction process of Boolean matrix $\mathcal{M}$.

We now analyze the complexity of Algorithm computeHoms. The entry $\mathcal{D}(u, v)$ of function BottomUpTraversal is checked once for every pair of nodes $(u \in$ children $(p), v \in$ children $(q))$ (line 4$)$. The entry $\mathcal{D}(p$, $v)$ is checked once for every pair of nodes $(p \in P$, $v \in \operatorname{children}(q)$ ) (line 5). Therefore, the total number of times these two entries are checked is no more than $|P| \times|Q|$.

The entry $\mathcal{A}$ (parent of $p$, parent of $q$ ) in line 4 and the entry $\mathcal{A}(p$, parent of $q)$ in line 5 of function TopDownTraversal are checked once for every pair of nodes $(p \in P, q \in Q)$. The total number of times these two entries are checked is no more than $|P| \times|Q|$. Therefore, the time and memory complexities of Algorithm computeHoms are both $O(|P| \times|Q|)$.

During the candidate generation, we cannot, however, simply discard candidates that are redundant, since they may be needed for generating non-redundant patterns. For instance, the pattern $P_{5}$ shown in Fig. $3 \mathrm{~b}$ is redundant, but it is needed (as the left operand in a join operation with $P_{7}$ ) to generate the non-redundant pattern $P_{6}$ shown in the same figure. Clearly, we want to avoid as much as possible generating patterns that are redundant. In order to do so, we introduce the notion of expandable pattern.

Definition 1 (Expandable pattern) A pattern $P$ is expandable, if it does not have a redundant node $X$ such that: (1) $X$ is not on the rightmost path of $P$, or (2) $X$ is on the rightmost path of $P$ and $L_{X}$ is equal to $L_{X_{1}} \cup \ldots \cup L_{X_{k}}$, where $X_{1}, \ldots, X_{k}$ are the images of node $X$ under a homomorphism from $P$ to itself.

Based on Definition 1, if a pattern is not expandable, every expansion of it is redundant. Therefore, only expandable patterns in a class are considered for expansion.

\subsubsection{Expandable Pattern Refining}

The number of expandable patterns enumerated by the equivalence class expansion process can still be very large, particularly when the frequent patterns to find have both a high depth and a high branching factor. In order to further reduce the number of generated patterns, we present below a pattern refining method which exploits properties of the equivalence class-based pattern expansion. We observe that the specificity relation $\prec$ induces a linear order on 
patterns in a given equivalence class whose rightmost leaf nodes have the same label: for any two patterns $P_{x}^{i}$ and $P_{x}^{j}$ in the equivalence class $[P], P_{x}^{i} \prec P_{x}^{j}$ if $i>j$. Clearly, the occurrence set of $P_{x}^{i}(x)$ is a subset of the occurrence set of $P_{x}^{j}(x)$, for $i>j$.

Let $P_{1}, P_{2}, \ldots, P_{n}$ stand for a sequence of $n$ expandable patterns satisfying the above linear order. Each pattern in the sequence has a rightmost leaf node $x$. For a pattern $P_{k}$, if the occurrence set of $P_{k}(x)$ is the same as the union of the occurrence sets of $P_{i}(x), i=1, \ldots, k-1$, then the set of occurrences of $P_{k}$ is the same as the union of occurrence sets of $P_{i}$ 's. In this case, it is not useful to further expand $P_{k}$, since it is refined by a set of more specific patterns. We call $P_{k}$ a refinable pattern. In Fig. 7, pattern $Q_{2}$ is refined by $Q_{1}$.

In order to efficiently identify refinable patterns, we keep the patterns $P_{x}^{i}$ in each class sorted by the node label $x$ primarily and by the position $p$ (in descending order) secondarily. Figure 6 shows patterns of a class in sorted order. Given a sorted pattern list; the equivalence class expansion process considers ordered pairs of patterns in the class for expansion. This way, the candidate generation process outputs a new class list which is also sorted based on this order, and no explicit sorting is needed.

In the implementation, we scan patterns of a given class in descending order. For each pattern $P$ under consideration, we check whether it has a preceding pattern $Q$, such that the rightmost leaf nodes of $P$ and $Q$ have the same label and the same occurrence list. If it is the case, $P$ is a refinable pattern and is excluded from further expansion. The process is summarized in Procedure CheckClassElements shown in Fig. 5. Our experiments show that the pattern pruning technique can effectively reduce the pattern search space.

\subsubsection{Finding Maximal Patterns}

One way to compute the maximal patterns is to use a postprocessing pruning method. That is, first compute the set $S$ of all frequent homomorphic patterns, and then do the maximality check and eliminate non-maximal patterns by

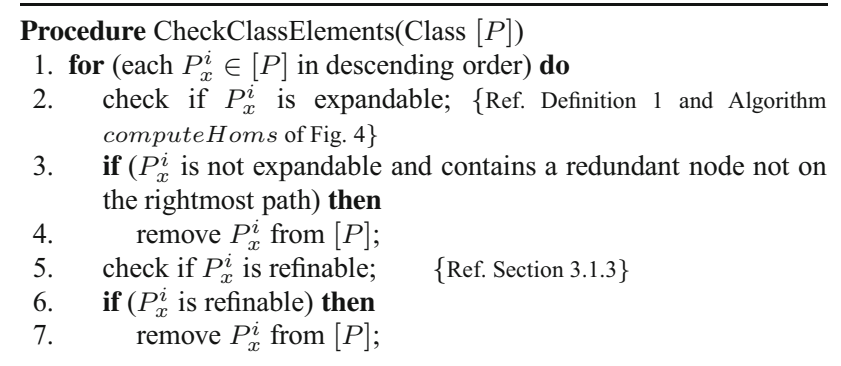

Fig. 5 Procedure CheckClassElements checking the specificity relation on every pair of patterns in $S$. However, the time complexity of this method is $\mathrm{O}\left(|S|^{2}\right)$. It is, therefore, inefficient since the size of $S$ can be exponentially larger than the number of maximal patterns.

We have developed a better method which can reduce the number of frequent patterns that need to go through the maximality check. During the course of mining frequent patterns, the method locates a subset of frequent patterns called locally maximal patterns. A pattern $P$ is locally maximal if it is frequent and there exists no frequent pattern in the class $[P]$. Clearly, a non-locally maximal pattern is not maximal. Then, in order to identify maximal patterns, we check only locally maximal patterns for maximality. Our experiments show that this improvement can dramatically reduce the number of frequent patterns checked for maximality.

\subsection{Support Computation}

Recall that the support of a pattern $P$ in the input data tree $T$ is defined as the size of the occurrence list $L_{R}$ of the root $R$ of $P$ on $T$ (Sect. 2). To compute $L_{R}$, a straightforward method is to first compute the relation $O C$ which stores the set of occurrences of $P$ under all possible homomorphisms of $P$ to $T$ and then "project" $O C$ on column $R$ to get $L_{R}$. Fortunately, we can do much better using a twig-join approach to compute $L_{R}$ without enumerating all homomorphisms of $P$ to $T$. Our approach for support computation is a complete departure from existing approaches.

A holistic twig-join approach In order to compute $L_{X}$, we exploit a holistic twig-join approach (e.g., TwigStack [4]), the state-of-the-art technique for evaluating tree pattern queries on tree data. Algorithm TwigStack works in two phases. In the first phase, it computes the matches of the individual root-to-leaf paths of the pattern. In the second phase, it merge-joins the path matches to compute the results for the pattern. TwigStack ensures that each solution to each individual query root-to-leaf path is guaranteed to be merge-joinable with at least one solution of each of the other root-to-leaf paths in the pattern. Therefore, the algorithm can guarantee worst-case performance linear to the size of the data tree inverted lists (the input) and the size of the pattern matches in the data tree (the output), i.e., the algorithm is optimal.

By exploiting the above property of TwigStack, we can compute the support of $P$ at the first phase of TwigStack when it finds data nodes participating in matches of root-toleaf paths of $P$. There is no need to enumerate the occurrences of pattern $P$ on $T$ (i.e., to compute the occurrence relation $O C$ ).

The time complexity of the above support computation method is $O(|P| \times|T|)$, where $|P|$ and $|T|$ denote the size of 


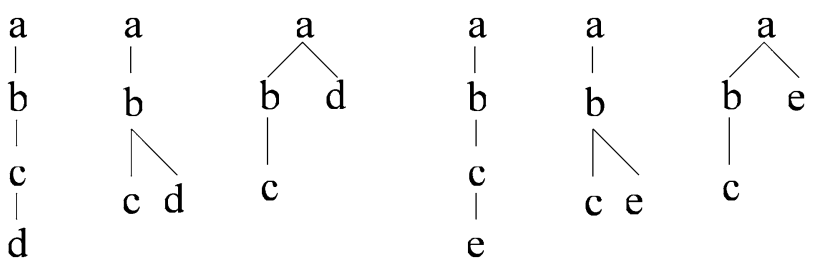

Fig. 6 Sorted patterns in class $[a / b / c]$

pattern $P$ and of the input data tree $T$, respectively. Its space complexity is the $\min (|T|,|P| \times$ heigh $(T))$. We note that, on the other hand, the problem of computing an unordered embedding from $P$ to $T$ is NP-complete [12]. As a consequence, a state-of-the-art unordered embedded pattern mining algorithm Sleuth [26] computes pattern support in $O\left(|P| \times|T|^{2|P|}\right)$ time and $O\left(|P| \times|T|^{|P|}\right)$ space.

Nevertheless, the TwigStack-based method can still be expensive for computing the support of a large number of candidates, since it needs to scan fully the inverted lists corresponding to every candidate pattern. We present below an incremental method, which computes the support of a pattern $P$ by leveraging the computation done at its parent patterns in the search space.

Computing occurrence lists incrementally Let $P$ be a pattern and $X$ be a node in $P$ labeled by $a$. Using TwigStack, $P$ is computed by iterating over the inverted lists corresponding to every pattern node. If there is a sublist, say $L_{X}$, of $L_{a}$ such that $P$ can be computed on $T$ using $L_{X}$ instead of $L_{a}$, we say that node $X$ can be computed using $L_{X}$ on $T$. Since $L_{X}$ is non-strictly smaller than $L_{a}$, the computation cost can be reduced. Based on this idea, we propose an incremental method that uses the occurrence lists of the two parent patterns of a given pattern $P$ to compute $P$.

Let pattern $Q$ be a join outcome of $P_{x}^{i} \otimes P_{y}^{j}$. By the definition of the join operation, we can easily identify a homomorphism from each parent $P_{x}^{i}$ and $P_{y}^{j}$ to $Q$.

Proposition 2 Let $X^{\prime}$ be a node in a parent $Q^{\prime}$ of $Q$ and $X$ be the image of $X^{\prime}$ under a homomorphism from $Q^{\prime}$ to $Q$. The occurrence list $L_{X}$ of $X$ on $T$ is a sublist of the occurrence list $L_{X^{\prime}}$ of $X^{\prime}$ on $T$.

Sublist $L_{X}$ is the inverted list of data tree nodes that participate in the occurrences of $Q$ to $T$. By Proposition 2, $X$ can be computed using $L_{X^{\prime}}$ instead of using the corresponding label inverted list. Further, if $X$ is the image of nodes $X_{1}$ and $X_{2}$ defined by the homomorphisms from the left and right parent of $Q$, respectively, we can compute $X$ using the intersection, $L_{X_{1}} \cap L_{X_{2}}$, of $L_{X_{1}}$ and $L_{X_{2}}$ which is the sublist of $L_{X_{1}}$ and $L_{X_{2}}$ comprising the nodes that appear in both $L_{X_{1}}$ and $L_{X_{2}}$.
Using Proposition 2, we can compute $Q$ using only the occurrence list sets of its parents. Thus, we only need to store with each frequent pattern its occurrence list set. Our method is space efficient since the occurrence lists can encode in linear space an exponential number of occurrences for the pattern [4]. In contrast, the state-of-the-art methods for mining embedded patterns [26, 27] have to store information about all the occurrences of each given pattern in $T$.

Occurrence lists as bitmaps The occurrence list $L_{X}$ of a pattern node $X$ labeled by $a$ on $T$ can be represented by a bitmap on $L_{a}$. This is a bit array of size $\left|L_{a}\right|$ which has a "1" bit at position $i$ iff $L_{X}$ comprises the tree node at position $i$ of $L_{a}$. Then, the occurrence list set of a pattern is the set of bitmaps of the occurrence lists of its nodes. Figure $2 \mathrm{c}$ shows an example of bitmaps for pattern occurrence lists.

As verified by our experimental evaluation, storing the occurrence lists of multiple patterns as bitmaps results in important space savings. Bitmaps offer CPU cost saving as well by allowing the translation of pattern evaluation to bitwise operations. This bitmap technique is initially introduced and exploited in [20,21, 23, 24] for materializing tree pattern views and for efficiently answering queries using materialized views.

Example 1 Figure 7 shows an example of the incremental method for computing the support of $Q_{1}$ and $Q_{2}$, the two outcomes of $P_{1} \otimes P_{2}$ on the data tree $T$ of Fig. 2a. We assume minsup is one. Each node of the patterns $P_{1}$ and $P_{2}$ is associated with its occurrence list together with the corresponding bitmap vector. To compute $Q_{1}$ and $Q_{2}$, the bitmaps of $P_{1}$ and $P_{2}$ are ANDed and the resulting bitmaps are attached to nodes of $Q_{1}$ and $Q_{2}$. These bitmaps are used as input to compute $Q_{1}$ and $Q_{2}$ using TwigStack. The bitmap output associated with each pattern node indicates the occurrence list of that node on $T$. Note that pattern $Q_{2}$ is refined by $Q_{1}$ and thus will not be further expanded.

\subsection{The Tree Pattern Mining Algorithm}

We present now our homomorphic tree pattern mining algorithm called HomTreeMiner (Fig. 8). The first part of the algorithm computes the sets containing all frequent 1patterns $F_{1}$ (i.e., nodes) and 2-patterns $F_{2}$ (lines 1-2). $F_{1}$ can be easily obtained by finding inverted lists of $T$ whose size (in terms of number of nodes) is no less than minsup. The total time for this step is $O(|T|) . F_{2}$ is computed by the following procedure: Let $X / Y$ denote a 2-pattern formed by two elements $X$ and $Y$ of $F_{1}$. The support of $X / Y$ is computed via algorithm TwigStack on the inverted lists $L_{l b(X)}$ and $L_{l b(Y)}$ that are associated with labels $l b(X)$ and 

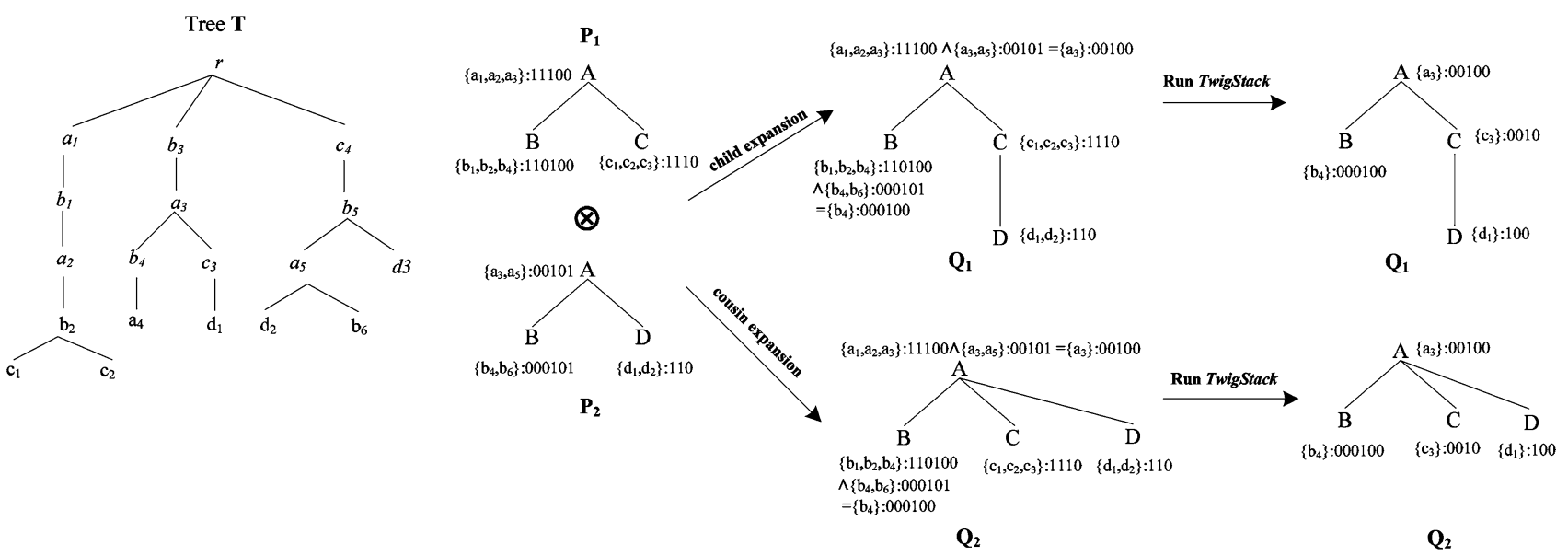

Fig. 7 An example of incremental support computation for the outcomes of $P_{1} \otimes P_{2}$ on tree $T$

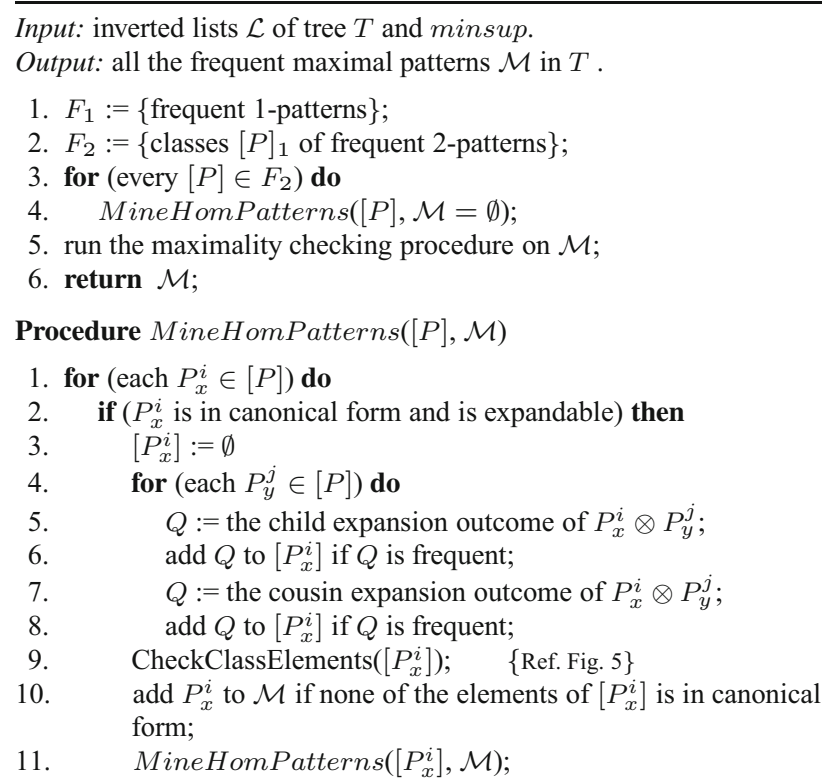

Fig. 8 Homomorphic tree pattern mining algorithm

$l b(Y)$, respectively. The total time for each 2-pattern candidate is $O(|T|)$.

The main part of the computation is performed by procedure MineHomPatterns which is invoked for every frequent 2-pattern (Lines 3-4). This is a recursive procedure. It tries to join every $P_{x}^{i} \in[P]$ with any other element $P_{y}^{j} \in[P]$ including $P_{x}^{i}$ itself. Then, it computes the support of the child expansion and the cousin expansion outcomes in that order and adds them to $\left[P_{x}^{i}\right]$ if they are frequent (Lines 1-8). Once all $P_{y}^{j}$ have been processed, Procedure CheckClassElements of Fig. 5 is invoked on class $\left[P_{x}^{i}\right]$ (Line 9). Subsequently, the algorithm checks whether $P_{x}^{i}$ is a locally maximal pattern. If so, $P_{x}^{i}$ is added to the maximal pattern set $\mathcal{M}$ (Line 10). Then, the new class $\left[P_{x}^{i}\right]$ is recursively explored in a depth-first manner (Line 11). The recursive process is repeated until no more frequent patterns can be generated.

Once all the locally maximal patterns have been found, the maximality check procedure described in Sect. 3.1 is run to identify maximal patterns among the locally maximal ones and the results are returned to the user (Lines 5-6).

Before expanding a class $[P]$, we make sure that $P$ is expandable and is in canonical form (line 2 in MineHomPatterns). Our approach is independent of any particular canonical form; it can work with any systematic way of choosing a representative from isomorphic representations of the given pattern, such as those presented in $[7,26]$. Efficient methods for checking canonicity can also be drawn from [7, 26].

Complexity The total cost for generating a new class $\left[P_{x}^{i}\right]$ is $O\left(n^{2} \times|T| \times|P|\right)$, where $n$ is the number of elements of $[P]$. In terms of memory consumption, observe that the algorithm only needs to load in memory the classes along a path in a depth-first traversal of the search space. In fact, it only needs to store in memory occurrence lists for two classes at a time: the current class $[P]$ and a new class $\left[P_{x}^{i}\right]$. Since occurrence lists of each pattern in a class are materialized as bitmaps, the memory footprint of the algorithm is very small. This is verified by our experimental results presented in Sect. 4 .

\section{Experimental Evaluation}

We implemented our algorithm HomTreeMiner and we conducted experiments to: (a) compare the features of the extracted (maximal) homomorphic patterns with those of (maximal) embedded patterns and (b) study the performance of HomTreeMiner in terms of execution time, memory consumption and scalability. To evaluate the 
Table 1 Dataset statistics

\begin{tabular}{lrrll}
\hline Dataset & Tot. \#nodes & \#labels & Max/avg depth & \#paths \\
\hline Treebank & $2,437,666$ & 250 & $36 / 8.4$ & $1,392,231$ \\
XMark & 83,533 & 74 & $12 / 5.6$ & 60,853 \\
CSlogs & 772,188 & 13,355 & $86 / 4.4$ & 59,691 (\#trees) \\
\hline
\end{tabular}

effect of the pattern refining technique described in Sect. 3.1.3, we consider also a basic version of HomTreeMiner that does not employ that optimization in its mining process. That basic version was introduced in [22] and is called HomTMBasic in the following paragraphs.

To the best of our knowledge, there is no previous algorithm computing homomorphic patterns from data trees. Therefore, we compared the performance of our algorithm with state-of-the-art algorithms that compute embedded patterns on the same dataset.

Our implementation was coded in Java. All the experiments reported here were performed on a workstation equipped with an Intel Xeon CPU 3565 @3.20 GHz processor with 8 GB memory running JVM 1.7.0 on Windows 7 Professional. The Java virtual machine memory size was set to the default $4 \mathrm{~GB}$.

Datasets We have ran experiments on three real and benchmark datasets with different structural properties. Their main characteristics are summarized in Table 1.

Treebank $^{1}$ is a real XML dataset derived from computation linguistics. It models the syntactic structure of English text and provides a hierarchical representation of the sentences in the text by breaking them into syntactic units based on part of speech. The dataset is deep and comprises highly recursive and irregular structures.

$X$ Mark $^{2}$ is an XML benchmark dataset generated using the data generator with factor $=0.05$. It is deep and has many regular structural patterns. It includes very few recursive elements.

CSlogs $s^{3}$ is a real dataset and is composed of users' access trees to the CS department Web site at RPI. The dataset contains 59,691 trees that cover a total of 13,355 unique web pages. The average size of each tree is 12.94 .

\subsection{Algorithm Performance}

We compare the performance of HomTreeMiner with two unordered embedded tree mining algorithms Sleuth [26] and EmbTreeMiner [19]. Sleuth was designed to mine embedded patterns from a set of small trees. In order to

\footnotetext{
${ }^{1}$ http://www.cis.upenn.edu/ treebank.

2 http://monetdb.cwi.nl/xml/.

${ }^{3}$ http://www.cs.rpi.edu/ zaki/software/.
}

allow the comparison in the single large tree setting, we adapted Sleuth by having it return as support of a pattern the number of its root occurrences in the data tree. EmbTreeMiner is a newer embedded tree mining algorithm which, as HomTreeMiner, exploits the twig-join approach and bitmaps to compute pattern support.

To the best of our knowledge, direct mining of maximal embedded patterns has not been studied in the literature. We therefore use post-processing pruning which eliminates non-maximal patterns after computing all frequent embedded patterns. For this task, we implemented the unordered tree inclusion algorithm described in [12]. As our experiments show, the cost of this post-processing step is in general not significant compared to the frequent pattern mining cost.

To allow Sleuth - which is slower-to extract some patterns within a reasonable amount of time, we used a fraction of the Treebank dataset which consists of $35 \%$ of the nodes of the original tree. We measured execution times over the entire Treebank dataset in the scalability experiment.

Candidate pattern generated Figs. 9c, 10c and 11c compare the total candidates generated by sleuth, EmbTreeMiner, HomTMBasic and HomTreeMiner, respectively, under different support thresholds on the Treebank, XMark and CSlogs datasets.

As one can see, the search space of a homomorphic pattern mining can be larger than that of embedded pattern mining for low support levels. On Treebank, for instance, HomTreeMiner computes 17 times more candidates than EmbTreeMiner at minsup $=30 \mathrm{k}$. Since Treebank contains many deep, highly recursive paths, the search space of homomorphic patterns becomes substantially large at low support levels. Like Treebank, XMark has many deep paths, and therefore, the search space of homomorphic patterns becomes large at low support levels. For example, on XMark at minsup $=700$, HomTreeMiner generates about 2.23 times more candidates than EmbTreeMiner. The number of candidates generated by HomTreeMiner and EmbTreeMiner is comparable on CSlogs. The difference in the number of candidates generated by sleuth and EmbTreeMiner is not noticeable on all the testing cases.

We notice that HomTMBasic generates substantially more candidates than HomTreeMiner for low support levels. For instance, on XMark at minsup $=650$, HomTMBasic generates about 9 times more candidates than HomTreeMiner. On CSlogs at minsup $=250$, HomTMBasic generates about 5 times more candidates than HomTreeMiner. This indicates that the pattern refining technique enables HomTreeMiner to reduce substantially the search space when it is applicable.

Execution time We measure the total elapsed time for producing maximal frequent patterns at different support 


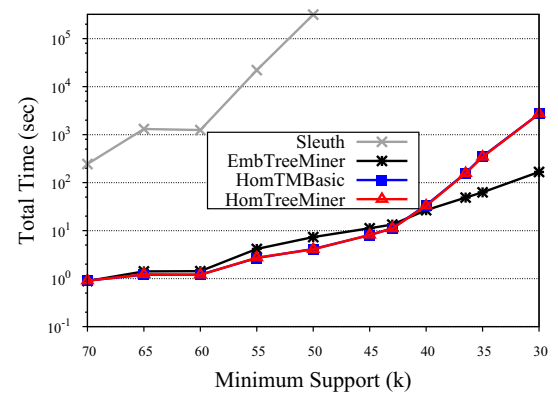

(a)

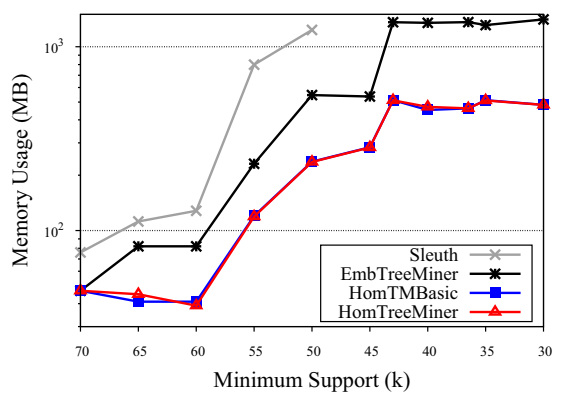

(b)

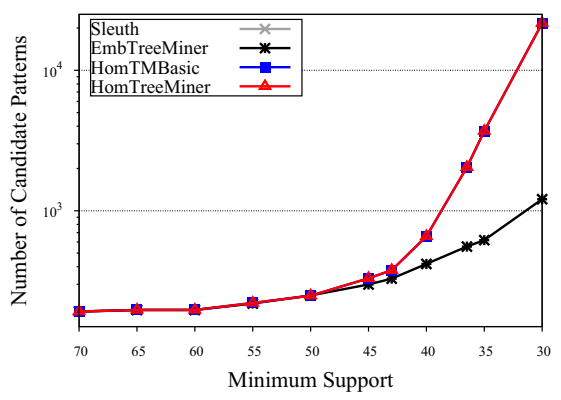

(c)

Fig. 9 Performance comparison on a fraction of treebank. a Run time versus support. b Memory usage. c Candidate patterns

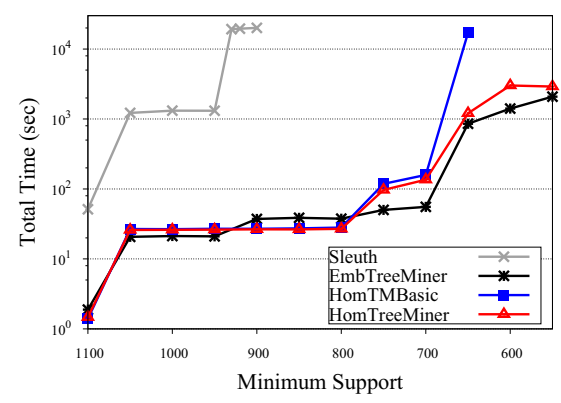

(a)

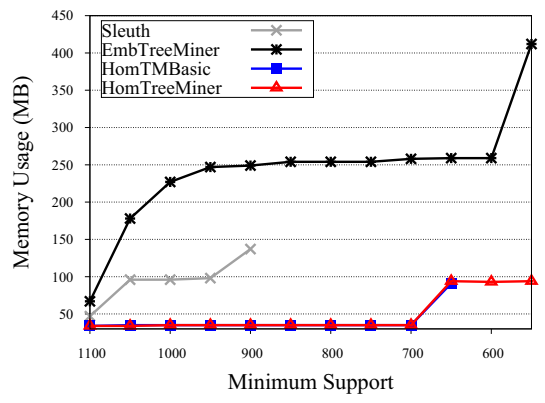

(b)

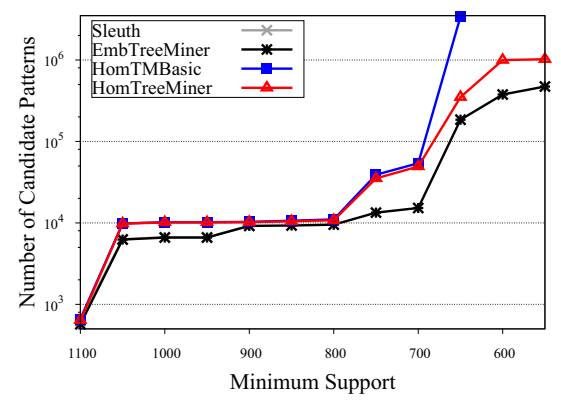

(c)

Fig. 10 Performance comparison on XMark. a Run time versus support. b Memory usage. c Candidate patterns

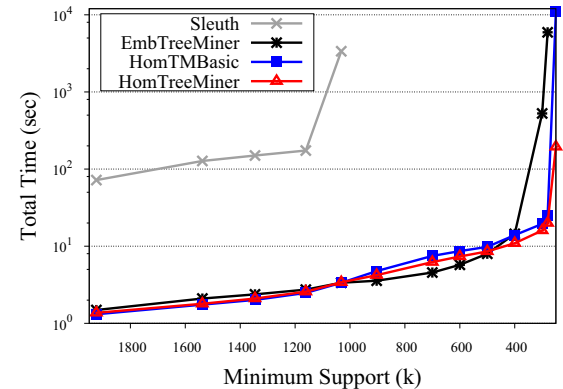

(a)

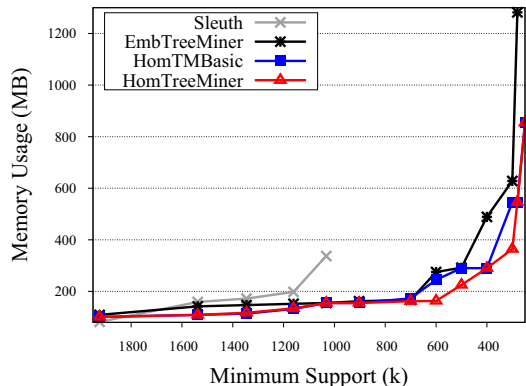

(b)

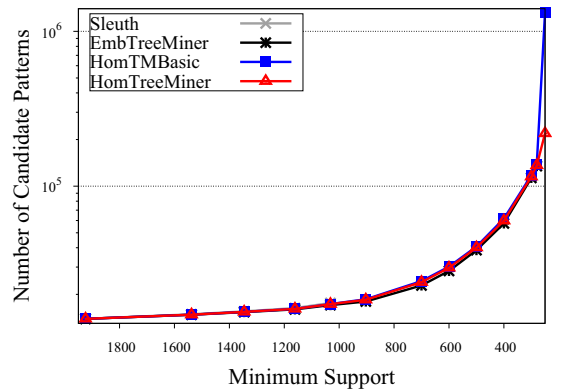

(c)

Fig. 11 Performance comparison on CSlogs. a Run time versus support. b Memory usage. c Candidate patterns

thresholds. The total time involves the time to generate candidate patterns, compute pattern support and check maximality of frequent patterns.

Figures 9a, 10a and 11a compare the total elapsed time of the four algorithms under different support thresholds on the Treebank, XMark and CSlogs datasets. Due to prohibitively long times, we stopped testing Sleuth when support levels are below certain values on each dataset.

We can see that HomTreeMiner runs orders of magnitude faster than Sleuth, especially for low support levels. The rate of increase of the running time for HomTreeMiner is slower than that for Sleuth as the support level decreases. This is expected, since HomTreeMiner computes the support of a homomorphic pattern in time linear to the input data size, whereas this computation is exponential for embedded pattern miners (Sect. 3.2). Furthermore, Sleuth has to keep track of all possible embedded occurrences of a candidate to a data tree and to perform expensive join operations over these occurrences.

The large number of candidate homomorphic patterns can negatively affect the time performance of HomTreeMiner at low support levels. For instance, on Treebank, HomTreeMiner shows similar or better performance than EmbTreeMiner when support levels are above $40 \mathrm{~K}$ and both generate a similar number of candidates. When minsup decreases below $40 \mathrm{~K}$, the execution time of HomTreeMiner increases noticeably faster than that of EmbTreeMiner due to the substantially larger number of 
candidates evaluated by HomTreeMiner. At minsup $=30 \mathrm{k}$, in order to evaluate 17 times more candidates, HomTreeMiner runs about 15 times slower than EmbTreeMiner.

However, even though the number of (candidate and frequent) homomorphic patterns is always larger than the number of embedded patterns, this difference is not so pronounced in shallower datasets like CSlogs. As we can see from Fig. 11a, HomTreeMiner can largely outperform EmbTreeMiner at low support levels. This is due to its efficient computation of pattern support which does not require the enumeration of pattern occurrences and the embedding checking as is the case with EmbTreeMiner [19].

From the results, we observe that HomTreeMiner can largely outperform HomTMBasic, when it is able to substantially reduce the search space with the refinement technique. For instance, on XMark at minsup $=650$, HomTreeMiner runs more than 13 times faster than HomTMBasic.

Memory usage We measured the memory footprint of the four algorithms with varying support thresholds. The results are shown in Figs. 9b, 10b and 11b. We can see that HomTreeMiner always has the best memory performance. It consumes substantially less memory than both Sleuth and EmbTreeMiner in all the test cases. This is mainly because Sleuth needs to enumerate and store in memory all the pattern occurrences for candidates under consideration. In contrast, HomTreeMiner avoids storing pattern occurrences by storing only bitmaps of occurrence lists which are usually of insignificant size. Although EmbTreeMiner does not store pattern occurrences, it still has to generate pattern occurrences as intermediate results, the size of which can be substantial at low support levels. The memory performance of HomTMBasic is similar to that of HomTreeMiner. The results indicate that the memory performance of a mining algorithm is mainly determined by its pattern support computation.

\subsection{Algorithm Scalability}

In our final experiment, we studied the scalability of the three algorithms EmbTreeMiner, HomTMBasic and HomTreeMiner as we increase the input data size. We omit the comparison with sleuth, since sleuth was unable to finish within a reasonable time even on the smallest size of input.

For Treebank, we generated ten fragments of increasing size and fixed minsup at $4.5 \%$. For XMark, we generated 10 XMark trees by setting factor $=0.01,0.02, \ldots, 0.1$ and fixed minsup at $1 \%$. For CSlogs, we generated 7 datasets of different sizes (from $40 \mathrm{k}$ trees and up to $100 \mathrm{k}$ ) by randomly choosing trees from the original CSlogs. We fixed minsup at 400.

The results show that HomTreeMiner has the best time performance on both XMark and CSlogs (Fig. 12b, c); it runs slightly slower than EmbTreeMiner on Treebank (Fig. 12a). The reason is that, on both XMark and CSlogs, the number of candidates evaluated by HomTreeMiner is similar to that by EmbTreeMiner, whereas on Treebank, it needs to evaluate $56 \%$ more candidates on average. On CSlogs, the growth of the running time of EmbTreeMiner becomes much sharper with datasets containing $80 \mathrm{k}$ trees and up. EmbTreeMiner is unable to finish within 5 hours on CSlogs containing $90 \mathrm{k}$ trees and up. HomTMBasic has similar time performance with HomTreeMiner on both Treebank and XMark. However, on CSlogs of size $90 \mathrm{k}$ and $100 \mathrm{k}$, HomTreeMiner outperforms HomTMBasic by a factor of more than 2.5. The reason is that, in these two cases, HomTMBasic has to evaluate about $47 \mathrm{k}$ more candidates in total and generates twice as many frequent patterns on average than HomTreeMiner.

Figure 13a-c show that HomTreeMiner always has the smallest memory footprint. The growth of its memory consumption is much slower than that of EmbTreeMiner.

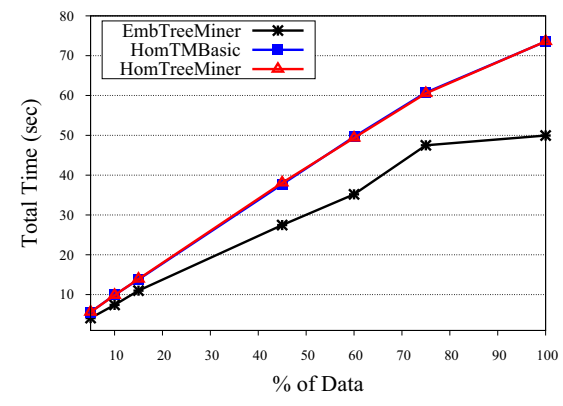

(a)

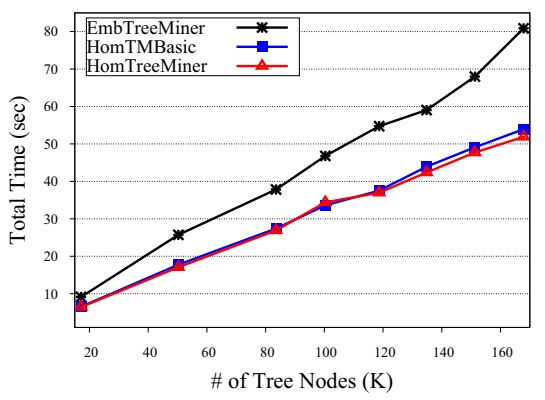

(b)

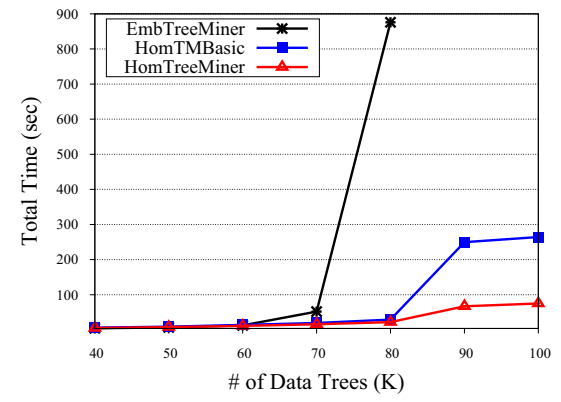

(c)

Fig. 12 Run time scalability comparison on the three datasets with increasing size. a Treebank $(\operatorname{minsup}=4.5 \%)$. b XMark $($ minsup $=1 \%)$. c CSlogs (minsup $=400)$ 


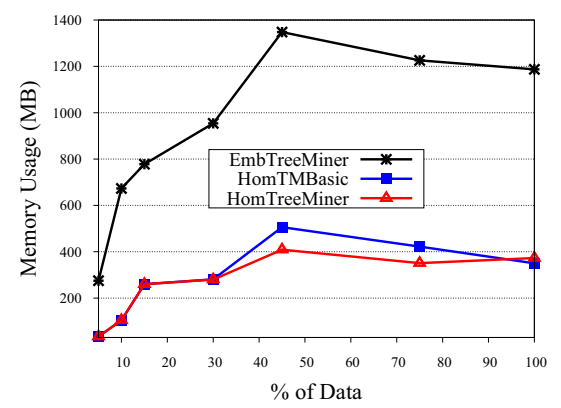

(a)

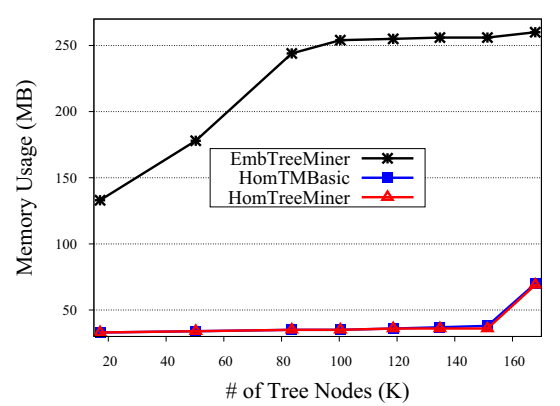

(b)

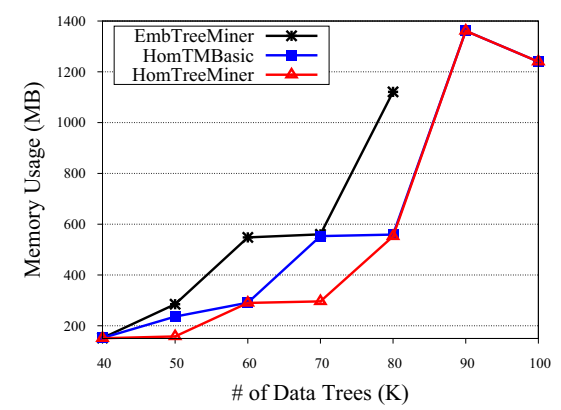

(c)

Fig. 13 Memory usage scalability comparison on the three datasets with increasing size. a Treebank $($ minsup $=4.5 \%)$. b XMark $($ minsup $=1 \%)$. c CSlogs $($ minsup $=400)$

Table 2 Statistics for maximal frequent patterns mined from the three datasets

\begin{tabular}{|c|c|c|c|c|c|c|c|c|c|c|}
\hline Dataset & Morphism & $\begin{array}{l}\text { \# freq. } \\
\text { patterns }\end{array}$ & $\begin{array}{l}\text { \# loc.max } \\
\text { patterns }\end{array}$ & $\begin{array}{l}\text { \# max. non. } \\
\text { red.patterns }\end{array}$ & $\begin{array}{l}\% \text { max. over } \\
\text { freq. patterns }\end{array}$ & $\begin{array}{l}\text { Average } \\
\text { \#nodes }\end{array}$ & $\begin{array}{l}\text { Average } \\
\text { height }\end{array}$ & $\begin{array}{l}\text { Average } \\
\text { fanout }\end{array}$ & $\begin{array}{l}\text { maximum } \\
\text { \#nodes }\end{array}$ & $\begin{array}{l}\text { \#common } \\
\text { max.patterns }\end{array}$ \\
\hline Treebank & Emb & 78 & $\mathrm{n} / \mathrm{a}$ & $2(8)$ & 2.6 & 0.63 & 0.375 & 0.25 & 3 & 1 \\
\hline$($ minsup $=35 \mathrm{k})$ & Hom & 521 & 158 & 9 & 1.7 & 5 & 2.11 & 2.11 & 8 & \\
\hline Treebank & Emb & 175 & $\mathrm{n} / \mathrm{a}$ & $13(32)$ & 7.4 & 1.47 & 0.66 & 0.78 & 5 & 5 \\
\hline$($ minsup $=30 \mathrm{k})$ & Hom & 2937 & 915 & 35 & 1.2 & 6.14 & 2.23 & 2.57 & 9 & \\
\hline XMark & Emb & 934 & $\mathrm{n} / \mathrm{a}$ & $14(19)$ & 1.5 & 2.63 & 1.05 & 1.58 & 5 & 6 \\
\hline$($ minsup $=800)$ & Hom & 853 & 26 & 15 & 1.76 & 4.67 & 1.93 & 2.6 & 10 & \\
\hline XMark & Emb & 43,441 & $\mathrm{n} / \mathrm{a}$ & $27(54)$ & 0.06 & 3.33 & 1 & 2.09 & 15 & 14 \\
\hline$($ minsup $=550)$ & Hom & 56,160 & 302 & 35 & 0.06 & 8.74 & 2.29 & 5.71 & 15 & \\
\hline CSlogs & Emb & 638 & $\mathrm{n} / \mathrm{a}$ & $133(164)$ & 20.8 & 2 & 0.896 & 1.1 & 5 & 119 \\
\hline$($ minsup $=400)$ & Hom & 816 & 307 & 152 & 18.6 & 2.53 & 1.11 & 1.41 & 6 & \\
\hline CSlogs & Emb & 2192 & $\mathrm{n} / \mathrm{a}$ & $250(375)$ & 11.4 & 1.68 & 0.728 & 0.95 & 6 & 192 \\
\hline$($ minsup $=280)$ & Hom & 1625 & 676 & 312 & 19.2 & 2.8 & 1.22 & 1.57 & 6 & \\
\hline
\end{tabular}

\subsection{Comparison of Mined Maximal Homomorphic and Embedded Patterns}

We computed different statistics on frequent and maximal frequent patterns mined by HomTreeMiner and EmbTreeMiner from the three datasets varying the support; the results are summarized in Table 2. For the comparison, we considered only maximal embedded patterns that contain no redundant nodes. We show the total number of maximal embedded patterns in parenthesis in Column 5. We can make the following observations.

First, HomTreeMiner is able to discover larger patterns than EmbTreeMiner for the same support level. As one can see in Table 2, the maximum size of frequent homomorphic patterns and the maximum size and average number of nodes, height and fanout of maximum frequent homomorphic patterns is never smaller (substantially larger in many cases) than that of the embedded patterns for the same support level.
Second, the number of homomorphic and embedded frequent patterns is substantially reduced if only maximal patterns are selected (Column 6 of Table 2). However, the effect is larger on homomorphic patterns as the number of frequent homomorphic patterns is usually larger than that of embedded patterns for the same support level (Column 3 of Table 2).

Third, by further looking at the mined maximal patterns, we find that the embedded maximal patterns at a certain support level can be partitioned into sets which correspond one-to-one to the maximal homomorphic patterns at the same support level so that all the embedded patterns in a set are less specific than the corresponding homomorphic pattern. Figure 14 shows, for each of the three datasets, examples of embedded maximal patterns each from the same set in the partition and the corresponding maximal homomorphic pattern. Therefore, for a number of applications, maximal homomorphic patterns can offer more information in a more compact way. 


\section{XMark:}

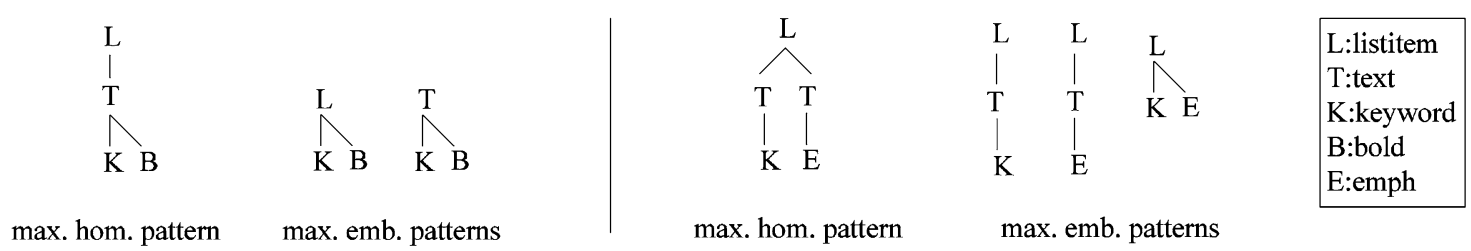

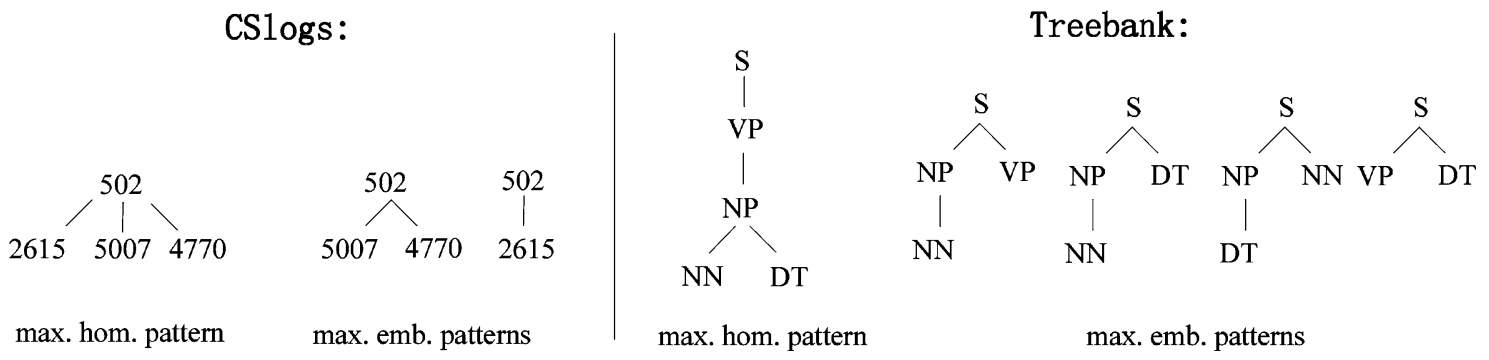

Fig. 14 Examples of maximal patterns mined from the three datasets

\section{Related Work}

We now discuss how our work relates to the existing literature. The problem of mining tree patterns from a set of small trees has been studied since the last decade. Among the many proposed algorithms $[2,3,5,6,8-11,14-19$, 25-27], only few mine unordered embedded patterns $[9,17,26]$.

Mining unordered embedded patterns TreeFinder [17] is the first unordered embedded tree pattern mining algorithm. It is a two-step algorithm. In the first step, it clusters the input trees by the co-occurrence of labels pairs. In the second step, it computes maximal trees that are common to all the trees of each cluster. A known limitation of TreeFinder is that it tends to miss many frequent patterns and is computationally expensive.

WTIMiner [9] transfers the frequent tree pattern mining to itemset mining. It first finds all the frequent itemsets, and then for each itemset found, it scans the database to count all the corresponding tree patterns. Although WTIMiner is complete, it is inefficient since the structural information is lost while mining for frequent itemsets. Further, the overhead for processing false positives may potentially reduce the performance.

Sleuth [26] extends the ordered embedded pattern mining algorithm TreeMiner [27]. Unlike TreeFinder, Sleuth uses the equivalence class pattern expansion method to generate candidates. To avoid repeated invocation of tree inclusion checking, Sleuth maintains a list of embedded occurrences with each pattern. It defines also a quadratic join operation over pattern occurrence lists to compute support for candidates. The join operation becomes inefficient when the size of pattern occurrence lists is large. Our approach relies on an incremental stack-based approach that exploits bitmaps to efficiently compute the support in time linear to the size of input data.

Mining maximal and closed induced patterns There exist algorithms [5, 18, 25] which focus on mining closed and maximal (induced) patterns. A frequent pattern $P$ is closed if none of $P$ 's proper superpatterns has the same support as that $P$ has; $P$ is maximal if none of $P$ 's proper superpatterns is frequent. The number of both maximal and closed patterns is usually much smaller, yet represents the same information as that of all frequent patterns. We below briefly mention about these algorithms.

CMTreeMiner [5] mines both closed and maximal frequent patterns from a set of small trees. Their method relies on a concept called blanket. The blanket of a pattern provides the set of immediate super patterns that are frequent. By comparing the occurrences of a given pattern with the occurrences of its blanket patterns, the algorithm determines whether the original pattern is closed or not. It uses pruning and heuristic techniques to reduce the search space. CMTreeMiner is the first algorithm which directly mines closed and maximal patterns without first generating all frequent patterns. However, it mines only for induced patterns; extending it to embedded patterns is not trivial.

PathJoin [25] finds maximal unordered induced patterns from a set of small trees. PathJoin assumes that no two siblings in data trees have the same label. It first discovers the set of maximal frequent paths and then it finds the tree patterns by joining the paths. After obtaining all frequent patterns, PathJoin keeps only maximal patterns by using a post-processing pruning, which eliminates those that are not maximal. Such a strategy will suffer from a significant overhead if the number of false positive paths is very high.

DryadeParent [18] mines closed induced patterns from a set of small trees. Observing that the performances of 
existing algorithms are dramatically affected by the branching factor of the tree patterns to find, DryadeParent makes the assumption that no two siblings in the data trees can have the same label (similar to PathJoin). The method first computes a set of tiles, which are closed frequent patterns of depth 1 . Then, it develops a hooking strategy that reconstructs the closed frequent patterns from these tiles. Similar to PathJoin, DryadeParent is designed based on the assumption that no two siblings in the data trees can have the same label. While this simplifies the problem, it limits the usage of the method in real applications.

The work on mining tree patterns in a single large tree or graph setting has so far been very limited. The only known papers are $[8,10,11]$ which focus on mining tree patterns with only child edges from a single graph and [19] which leverages homomorphisms to mine embedded tree patterns from a single tree. To the best of our knowledge, our work is the first one for efficiently mining (maximal) homomorphic tree patterns with descendant edges from a single large tree.

A preliminary version of algorithm HomTreeMiner was presented in [22]. The algorithm described in the present paper extends the previous version with an optimization technique which exploits the specificity relation to prune the space of candidate homomorphic patterns. The performance of the new version of HomTreeMiner is compared with that of its old version in the experimental section.

\section{Conclusion}

In this paper, we have addressed the problem of mining maximal frequent homomorphic tree patterns from a single large tree. We have provided a novel definition of maximal homomorphic patterns which takes into account homomorphisms, pattern specificity and the single tree setting. We have designed an efficient algorithm that discovers all frequent non-redundant maximal homomorphic tree patterns. Our approach employs an incremental stack-based frequency computation method that avoids the costly enumeration of all pattern occurrences required by previous approaches. An originality of our method is that matching information of already computed patterns is materialized as bitmaps, which greatly reduces both memory consumption and computation costs. An optimization technique further prunes the search space of candidate patterns. We have conducted extensive experiments to compare our approach with tree mining algorithms that mine embedded patterns when applied to a large data tree. Our results show that maximal homomorphic patterns are fewer and larger than maximal embedded tree patterns. Further, our algorithm is as fast as the state-of- the-art algorithm mining embedded trees from a single tree while outperforming it in terms of memory consumption and scalability.

Several applications are interested in extracting not all the frequent patterns, but only those that comply with a number of restrictions. We are currently working on incorporating user-specified constraints to the proposed approach to enable constraint-based homomorphic pattern mining.

Open Access This article is distributed under the terms of the Creative Commons Attribution 4.0 International License (http://crea tivecommons.org/licenses/by/4.0/), which permits unrestricted use, distribution, and reproduction in any medium, provided you give appropriate credit to the original author(s) and the source, provide a link to the Creative Commons license, and indicate if changes were made.

\section{References}

1. Amer-Yahia S, Cho S, Lakshmanan LVS, and Srivastava D (2001) Minimization of tree pattern queries. In: SIGMOD, pp 497-508

2. Asai T, Abe K, Kawasoe S, Arimura H, Sakamoto H, and Arikawa $S$ (2002) Efficient substructure discovery from large semistructured data. In: SDM, pp 158-174

3. Asai T, Arimura H, Uno T, Nakano S-I (2003) Discovering frequent substructures in large unordered trees. In: Discovery, Science, pp 47-61

4. Bruno N, Koudas N, and Srivastava D (2002) Holistic twig joins: optimal XML pattern matching. In: SIGMOD, pp 310-321

5. Chi Y, Xia Y, Yang Y, Muntz RR (2005) Mining closed and maximal frequent subtrees from databases of labeled rooted trees. IEEE Trans Knowl Data Eng 17(2):190-202

6. Chi Y, Yang Y, and Muntz RR (2004) Hybridtreeminer: an efficient algorithm for mining frequent rooted trees and free trees using canonical form. In: SSDBM, pp 11-20

7. Chi Y, Yang Y, Muntz RR (2005) Canonical forms for labelled trees and their applications in frequent subtree mining. Knowl Inf Syst 8(2):203-234

8. Dries A, Nijssen S (2012) Mining patterns in networks using homomorphism. In: SDM, pp 260-271

9. Feng Z, Hsu W, and Lee M-L (2005) Efficient pattern discovery for semistructured data. In: ICTAI, pp 294-301

10. Goethals B, Hoekx E, and den Bussche JV (2005) Mining tree queries in a graph. In: KDD, pp 61-69

11. Kibriya AM, Ramon J (2013) Nearly exact mining of frequent trees in large networks. Data Min Knowl Discov 27(3):478-504

12. Kilpeläinen P, Mannila H (1995) Ordered and unordered tree inclusion. SIAM J Comput 24(2):340-356

13. Miklau G, Suciu D (2004) Containment and equivalence for a fragment of xpath. J ACM 51(1):2-45

14. Nijssen S, Kok JN (2004) A quickstart in frequent structure mining can make a difference. In: KDD, pp 647-652

15. Tan H, Hadzic F, Dillon TS, Chang E, Feng L (2008) Tree model guided candidate generation for mining frequent subtrees from xml documents. TKDD 2(2):1-43

16. Tatikonda S, Parthasarathy S, Kurç TM (2006) Trips and tides: new algorithms for tree mining. In: CIKM, pp 455-464

17. Termier A, Rousset M-C, Sebag M (2002) Treefinder: a first step towards xml data mining. In: ICDM, pp 450-457 
18. Termier A, Rousset M-C, Sebag M, Ohara K, Washio T, Motoda H (2008) Dryadeparent, an efficient and robust closed attribute tree mining algorithm. IEEE Trans Knowl Data Eng 20(3):300-320

19. Wu X, Theodoratos D (2015) Leveraging homomorphisms and bitmaps to enable the mining of embedded patterns from large data trees. In: DASFAA, pp 3-20

20. Wu X, Theodoratos D (2016) Template-based bitmap view selection for optimizing queries over tree data. Int J Coop Inf Syst 25(3): $1-28$

21. Wu X, Theodoratos D, Kementsietsidis A (2015) Configuring bitmap materialized views for optimizing XML queries. World Wide Web 18(3):607-632

22. Wu X, Theodoratos D, Peng Z (2016) Efficiently mining homomorphic patterns from large data trees. In: DASFAA, pp 180-196

23. Wu X, Theodoratos D, Wang WH (2009) Answering XML queries using materialized views revisited. In: CIKM, pp $475-484$
24. Wu X, Theodoratos D, Wang WH, Sellis T (2013) Optimizing XML queries: bitmapped materialized views vs. indexes. Inf Syst 38(6):863-884

25. Xiao Y, Yao J-F, Li Z, Dunham MH (2003) Efficient data mining for maximal frequent subtrees. In: ICDM, pp 379-386

26. Zaki MJ (2005) Efficiently mining frequent embedded unordered trees. Fundam Inform 66(1-2):33-52

27. Zaki MJ (2005) Efficiently mining frequent trees in a forest: algorithms and applications. IEEE Trans Knowl Data Eng 17(8):1021-1035

28. Zhu F, Qu Q, Lo D, Yan X, Han J, Yu PS (2011) Mining top-k large structural patterns in a massive network. PVLDB 4(11):807-818

29. Zhu F, Yan X, Han J, Yu PS, Cheng H (2007) Mining colossal frequent patterns by core pattern fusion. In: ICDE, pp 706-715 